\title{
Recognition, intervention, and management of antisocial behaviour and conduct disorders in children and young people: summary of NICE-SCIE guidance
}

\author{
Stephen Pilling director ${ }^{1}$ professor $^{2}$, Nick Gould consultant ${ }^{4}$ emeritus professor ${ }^{5}$ professor $^{6}$, Craig \\ Whittington associate director (clinical effectiveness); lead systematic reviewer ${ }^{1}$, Clare Taylor senior \\ editor $^{7}$, Stephen Scott professor ${ }^{8}$ consultant child and adolescent psychiatrist; head ${ }^{9}$ director $^{10}$, On \\ behalf of the Guideline Development Group

\begin{abstract}
${ }^{1}$ National Collaborating Centre for Mental Health, University College London, London WC1E 7HB, UK; ${ }^{2}$ Centre for Outcomes Research and Effectiveness, University College London, London WC1E 7HB, UK; ${ }^{3}$ Research Department of Clinical, Educational and Health Psychology, University College London, London WC1E 7HB, UK; ${ }^{4}$ Social Care Institute for Excellence, London SW1Y 5BH, UK; ${ }^{5}$ Department of Social and Policy Sciences, University of Bath, Bath BA2 7AY, UK; ${ }^{6}$ School of Human Services and Social Work, Griffith University, QLD 4131, Australia; ${ }^{7}$ National Collaborating UK; ${ }^{9}$ National Conduct Problems Clinic and National Adoption and Fostering Clinic, Maudsley Hospital, London BR3 3BX, UK; ${ }^{10} \mathrm{National}$ Academy for Parenting Research, King's College London, London SE5 8AF, UK
\end{abstract} \\ Centre for Mental Health, Royal College of Psychiatrists, London E1 8AA, UK; ${ }^{8}$ Institute of Psychiatry, King's College London, London SE5 8AF,
}

This is one of a series of $B M J$ summaries of new guidelines based on the best available evidence; they highlight important recommendations for clinical practice, especially where uncertainty or controversy exists.

Antisocial behaviour and conduct disorders (including oppositional defiant disorder and conduct disorder) are the most common mental and behavioural problems in children and young people globally, with the frequency increasing in Western countries. ${ }^{1}$ In the United Kingdom 5\% of mental and behavioural problems in children and young people ( $\leq 18$ years) meet criteria for a conduct disorder, as do almost $40 \%$ of looked-after children, children who have been abused, and those on child protection or safeguarding registers. ${ }^{2}$ Conduct disorders are strongly associated with poor performance at school, social isolation, substance misuse, and involvement with the criminal justice system. ${ }^{3}$ A large proportion of children and young people with a conduct disorder will go on to be antisocial adults with impoverished and destructive lifestyles, ${ }^{3}$ especially if the conduct problems develop early, ${ }^{4}$ and a large minority will be diagnosed with antisocial personality disorder. ${ }^{5}$ Antisocial behaviour and conduct disorders often coexist with other mental health problems, place a heavy personal and economic burden on individuals and society, ${ }^{6}$ and involve a wide range of health, social care, educational, and criminal justice services.

This article summarises the most recent recommendations from the National Institute for Health and Clinical Excellence (NICE) on recognising and managing antisocial behaviour and conduct disorders in children and young people. ${ }^{7}$ The guideline was developed jointly with the Social Care Institute for Excellence (SCIE).

\section{Recommendations}

NICE recommendations are based on systematic reviews of the best available evidence and explicit consideration of cost effectiveness. When minimal evidence is available, recommendations are based on the Guideline Development Group's experience and opinion of what constitutes good practice. Evidence levels for the recommendations are given in italic in square brackets.

\section{Working safely and effectively}

Health and social care professionals working with children and young people who present with behaviour suggestive of a conduct disorder, or who have a conduct disorder, should be trained and competent to work with children and young people of all levels of learning ability, cognitive capacity, emotional maturity, and development. [Based on the experience and opinion of the Guideline Development Group (GDG)]

\section{Selective prevention}

Offer classroom based emotional learning and problem solving programmes for children typically aged 3-7 years in schools where classes have a high proportion of children identified to 
be at risk of developing oppositional defiant disorder or conduct disorder resulting from any of the following factors:

- Low socioeconomic status

- Low school achievement

- Child abuse or parental conflict

- Separated or divorced parents

- Parental mental health or substance misuse problems

- Parental contact with the criminal justice system.

[Based on moderate to high quality evidence from randomised controlled trials and on the experience and opinion of the GDG]

Provide these programmes in a positive atmosphere and ensure the interventions are designed to:

- Increase children's awareness of their own and others' emotions

- Teach self control of arousal and behaviour

- Promote a positive self concept and good peer relations

- Develop children's problem solving skills.

Typically the programmes should consist of up to 30 classroom based sessions over the course of one school year. [Based on moderate to high quality evidence from randomised controlled trials and on the experience and opinion of the GDG]

\section{Initial assessment of children and young people with a possible conduct disorder}

Undertake an initial assessment for a suspected conduct disorder if parents or carers, healthcare or social care professionals, school or college, or peer group raise concerns about persistent antisocial behaviour. [Based on the experience and opinion of the $G D G]$

Consider using the strengths and difficulties questionnaire ${ }^{8}$ (completed by a parent, carer, or teacher) and assess for the following complicating factors:

- A coexisting mental health problem (for example, depression, post-traumatic stress disorder)

- A neurodevelopmental condition (in particular, attention-deficit/hyperactivity disorder and autism)

- A learning disability or difficulty

- Substance misuse.

[Based on adequate quality evidence from observational studies and on the experience and opinion of the GDG]

If there are complicating factors, refer the child or young person to specialist child and adolescent mental health services for a comprehensive assessment. [Based on the experience and opinion of the $G D G]$

If there are no complicating factors, consider direct referral for an intervention. [Based on the experience and opinion of the $G D G]$

\section{Comprehensive assessment}

Standard components of this should include asking about and assessing:

- Core conduct disorders symptoms, including:

-Patterns of negativistic, hostile, or defiant behaviour in children aged under 11 years

-Aggression to people and animals, destruction of property, deceitfulness or theft, and serious violations of rules in children aged over 11 years
- Current functioning at home, school, or college and with peers

- Parenting quality

- History of any past or current mental or physical health problems.

[Based on the experience and opinion of the GDG]

Assess for:

- The risks faced by the child or young person. If needed, develop a risk management plan for self neglect, exploitation by others, self harm or harm to others

- The presence or risk of physical, sexual, and emotional abuse in line with local protocols for the assessment and management of these problems.

[Based on the experience and opinion of the GDG]

Conduct a comprehensive assessment of parents or carers, covering:

- Positive and negative aspects of parenting - in particular, any use of coercive discipline

- The parent-child relationship

- Positive and negative adult relationships in the family, including domestic violence

- Parental wellbeing, encompassing mental health, substance misuse (including whether alcohol or drugs were used during pregnancy), and criminal behaviour.

[Based on the experience and opinion of the GDG]

\section{Parent training programmes}

Offer a group parent training programme to the parents of those aged 3-11 years who have or are at high risk of oppositional defiant disorder or conduct disorder or are in contact with the criminal justice system because of antisocial behaviour. [Based on moderate to high quality evidence from randomised controlled trials]

In these programmes, involve both parents if possible and if in the best interests of the child or young person; and ensure that the programmes:

- Typically have 10-12 parents in a group

- Are based on a social learning model, using modelling, rehearsal, and feedback to improve parenting skills

- Typically consist of 10-16 meetings, each lasting 90-120 minutes

- Adhere to the developer's manual (which should have been positively evaluated in a randomised controlled trial) and use all of the necessary materials to ensure consistent implementation of the programme.

[Based on moderate to high quality evidence from randomised controlled trials and on the experience and opinion of the GDG]

\section{Foster carer/guardian training programmes}

Offer a group foster carer/guardian training programme to foster carers and guardians of those aged 3-11 years who have or are at high risk of oppositional defiant disorder or conduct disorder or are in contact with the criminal justice system because of antisocial behaviour. [Based on limited high quality evidence from randomised controlled trials and on the experience and opinion of the GDG]

In these programmes, involve both of the foster carers or guardians if possible and if in the best interests of the child or young person; and ensure that the programmes: 
- Modify the intervention to take account of the care setting in which the child is living

- Typically have 8-12 foster carers or guardians in a group

- Are based on a social learning model using modelling, rehearsal, and feedback to improve parenting skills

- Typically consist of 12-16 meetings, each lasting 90 to 120 minutes

- Adhere to the developer's manual (which should have been positively evaluated in a randomised controlled trial) and use all of the necessary materials to ensure consistent implementation of the programme.

[Based on high quality evidence from randomised controlled trials and on the experience and opinion of the GDG]

\section{Child focused programmes}

Offer group social and cognitive problem solving programmes to those aged between 9 and 14 years at high risk of or with oppositional defiant disorder or conduct disorder or if they are in contact with the criminal justice system because of antisocial behaviour. [Based on low to moderate quality evidence from randomised controlled trials]

Adapt these group programmes to the children's or young people's developmental level and ensure that they:

- Are based on a cognitive behavioural problem solving model

- Use modelling, rehearsal, and feedback to improve skills

- Typically consist of 10-18 weekly meetings, each lasting two hours

- Adhere to the developer's manual (which should have been positively evaluated in a randomised controlled trial) and use all of the necessary materials to ensure consistent implementation of the programme.

[Based on low to moderate quality evidence from randomised controlled trials and on the experience and opinion of the GDG]

\section{Multimodal interventions}

Offer multimodal interventions (for example, multisystemic therapy, in which a designated professional provides intensive support to the young person and their family in the home, school, and community, with the aim of reducing their antisocial behaviour) to those aged 11-17 years for the treatment of conduct disorder. [Based on low to high quality evidence from randomised controlled trials]

In multimodal interventions, involve the child or young person and their parents and carers, and ensure that the interventions:

- Have an explicit and supportive focus on the family

- Are based on a social learning model with interventions provided at individual, family, school, criminal justice, and community levels

- Are provided by specially trained case managers

- Typically consist of three to four meetings a week for three to five months

- Adhere to the developer's manual (which should have been positively evaluated in a randomised controlled trial) and use all of the necessary materials to ensure consistent implementation of the programme.

[Based on low to high quality evidence from randomised controlled trials and on the experience and opinion of the GDG]

\section{Improving access to services}

Provide information about the services and interventions in the local care pathway, including the:

- Range and nature of the interventions provided

- Settings in which services are delivered

- Processes by which a child or young person moves through the pathway

- Means by which progress and outcomes are assessed

- Delivery of care in related health and social care services.

[Based on the experience and opinion of the GDG and on the evidence reviewed in the NICE guideline on common mental health disorders ${ }^{9}$ ]

\section{Overcoming barriers}

Accessing care for antisocial behaviour and conduct disorders can be a potential barrier. ${ }^{10}$ To overcome this, there first needs to be wider recognition by teachers, social workers, community workers, primary care staff, and parents that persistent antisocial behaviour does not just result from "naughty" or "difficult" character traits but often has psychological and biological causes and responds to effective treatment. ${ }^{11}$ The possibility of a conduct disorder should be considered, particularly in higher risk groups such as looked-after children, children subject to harsh parenting, and those with attention-deficit/hyperactivity disorder. Evidence based interventions, such as parent training programmes, need to be offered at times when parents can attend, including evenings and weekends, and be delivered by well trained staff who receive ongoing supervision. If the antisocial behaviour or conduct disorder is not responding to intervention, consider the mental health needs of parents. Finally, service delivery needs to be well coordinated across agencies, including youth offending teams.

The members of the Guideline Development Group were Stephen Scott (chair), Nick Gould (deputy chair), Stephen Pilling (facilitator), Craig Whittington (systematic reviewer), Beth Anderson, Benedict Anigbogu, Sara Barratt, Ruth Braidwood, Maria Brewster, Barbara Compitus, Moira Doolan, Peter Fonagy, Laura Gibbon, Naomi Glover, Bronwyn Harrison, Flora Kaminski, Daphne Keen, Paul McArdle, Paul Mitchell, Maryla Moulin, Rose Nieto-Hernandez, Melinda Smith, Sarah Stockton, Clare Taylor, Jenny Taylor, Philippa Williams, Tony Wootton, and Amina Yesufu-Udechuku.

Contributors: All authors contributed to the conception and drafting of this article and revising it critically. They have all approved the final version. SP is the guarantor.

Competing interests: All authors have completed the ICMJE unified disclosure form at www.icmje.org/coi_disclosure.pdf (available on request from the corresponding author) and declare: (1) SP, CW, and CT had support from the National Collaborating Centre for Mental Health (NCCMH) for the submitted work; (2) SP receives funding from NICE to support guideline development work at the NCCMH; (3) NG receives funding from the Social Care Institute for Excellence to support guideline development work with NICE; (4) no other relationships or activities that could appear to have influenced the submitted work.

Provenance and peer review: Commissioned; not externally peer reviewed.

1 Collishaw S, Maughan B, Goodman R, Pickles A. Time trends in adolescent mental health. J Child Psychol Psychiatry 2004;45:1350-62.

2 Green H, McGinnity A, Meltzer H, Ford T, Goodman R. Mental health of children and young people in Great Britain, 2004: summary report. Office for National Statistics, 2005. young people in Great Britain, 2004: summary report. Office for National Statistics, 2005 .
Fergusson DM, Horwood LJ, Ridder EM. Show me a child at seven: consequences of conduct problems in childhood for psychosocial functioning in adulthood. J Child Psychol Psychiatry 2005;46:837-49 


\section{Further information on the guidance}

Factors that prompted the development of this guideline, included (in healthcare services) a lack of knowledge and recognition of conduct disorders and limited awareness that antisocial behaviour is a mental health problem and can therefore be "treated." Less than a quarter of disorders and limited awareness that antisocial behaviour is a mental health problem and can therefore be "treated." Less than a quarter of
children and young people with a conduct disorder receive specialist assessment or treatment ${ }^{12}$ and while parent training programmes are reasonably well established, much of the help being offered is probably ineffective because few practitioners are adequately trained. ${ }^{13}$ Similar problems exist in social care services. Conduct disorders are heavily over-represented in those needing social care, particularly looked-after children and abused children on safeguarding registers. ${ }^{2}$ However, conduct disorders are often under-recognised and undertreated by social care services, and when interventions are offered, they may not be evidence based.

Antisocial behaviour and conduct disorders can have a considerable detrimental impact on a child or young person's education, ${ }^{3}$ but schools may be ill equipped to cope with this. A stronger liaison between schools and healthcare and social care services is therefore crucial for the guideline to be fully implemented and selective prevention strategies to be successful. One challenge in instigating a preventive programme is to identify which vulnerable groups would benefit from intervention. The guideline provides some advice on this, as outlined above. Even when a child or young person has developed a conduct disorder, the evidence suggests that continued intervention can prevent antisocial personality disorder (for which treatments are limited) from developing in adulthood. ${ }^{6}$

\section{Methods}

This guideline was developed by the National Collaborating Centre for Mental Health using NICE's guideline methods (http://publications nice.org.uk/the-guidelines-manual-pmg6). The guideline review process involved comprehensive and systematic literature searches to identify relevant evidence for the clinical and economic reviews, with critical appraisal of the quality of the identified evidence. The limited evidence base required the development and refinement of existing methods, including procedures for extrapolating from other datasets and the incorporation and adaptation of recommendations from other NICE guidelines. A multidisciplinary team of healthcare and social care professionals from psychiatry, psychology, paediatrics, general practice nursing, social work, and education as well as service user and carer representatives (the GDG), was established to review the evidence and develop the subsequent recommendations. The guideline then went through an external consultation with stakeholders. The GDG considered the stakeholders' comments, reanalysed the data where necessary, and modified the guideline as appropriate.

NICE has produced three different versions of the guideline: a full version; a summary version known as the "NICE-SCIE guideline"; and a version for children and young people with antisocial behaviour and conduct disorders, their parents and carers, and the public. All these versions, as well as a pathway, are available from the NICE website. Updates of the guideline will be produced as part of NICE's guideline development programme.

\section{Areas for future research}

The effectiveness of:

- Parent training programmes for conduct disorders in children and young people aged 12 years and over

- Booster interventions to maintain the benefits of treatment and prevent relapse after successful treatment for antisocial behaviour and conduct disorders

- Combining treatment for mental health problems in parents with treatment for antisocial behaviour and conduct disorders in their children

- Strategies for improving uptake of and engagement with interventions for antisocial behaviour and conduct disorders by parents and young people

- Classroom based interventions for antisocial behaviour and conduct disorders

4 Moffitt T. Life-course-persistent versus adolescence-limited antisocial behaviour: a 10-year research review and a research agenda. In: Cicchetti D, Cohen DJ, eds. Developmental psychopathology. Volume 3: risk, disorder, and adaptation. Wiley, 2006:570-98.

5 National Collaborating Centre for Mental Health. Antisocial personality disorder: treatment, management and prevention. British Psychological Society, Royal College of Psychiatrists, 2010.

6 Scott S, Knapp M, Henderson J, Maughan B. Financial cost of social exclusion: follow up study of antisocial children into adulthood. BMJ 2001:323:191.

7 National Institute for Health and Clinical Excellence. Antisocial behaviour and conduct disorders in children and young people: recognition, intervention and management. (Clinical guideline 158.) 2013. http://guidance.nice.org.uk/CG158.

8 Goodman R, Meltzer H, Bailey V. The strengths and difficulties questionnaire: a pilot study on the validity of the self-report version. Eur Child Adolesc Psychiatry 1998;7:125-30.

9 National Institute for Health and Clinical Excellence. Common mental health disorders: identification and pathways to care. (Clinical guideline 123.) 2011. http://guidance.nice. org.uk/CG123.
10 Barber AJ, Tischler VA, Healy E. Consumer satisfaction and child behaviour problems in child and adolescent mental health services. J Child Health Care 2006;10:9-21.

11 National Collaborating Centre for Mental Health. Antisocial behaviour and conduct disorders in children and young people: recognition, intervention and management. British Psychological Society, Royal College of Psychiatrists [forthcoming].

12 Vostanis P, Meltzer H, Goodman R, Ford T. Service utilisation by children with conduct disorders-findings from the GB National Study. Eur Child Adolesc Psychiatry 2003;12:231-8.

13 Scott S. Conduct disorders in children. BMJ 2007;334:646-7.

Cite this as: BMJ 2013;346:f1298

(c) BMJ Publishing Group Ltd 2013 